\title{
Situación del mercado del arroz en Costa Rica: una mirada a la realidad
}

\author{
The state of the rice market in Costa Rica: a look at reality
}

\author{
Yendry Sánchez Picado' \\ María Fernanda Vega Solano
}

Recibido: 21/10/2016.

Corregido: 12/10/2016.

Aceptado: 25/10/2017.

\begin{abstract}
Resumen: El artículo muestra una visión general de la situación del mercado del arroz en Costa Rica entre el periodo 2002 - 2013, caracterizando la agrocadena que engloba el mercado, desde su producción hasta la comercialización. Así mismo, desarrolla indicadores relevantes para determinar la posición del país en lo que respecta a la seguridad alimentaria, esto con base en datos facilitados por la Corporación Arrocera Nacional de Costa Rica (CONARROZ). Por último, se da una mirada al impacto del mercado arrocero en el sector socioeconómico y de tal manera, que permita conocer los esfuerzos hechos por el Estado en materia de políticas públicas para coadyuvar en su desarrollo.
\end{abstract}

Palabras Claves: seguridad alimentaria; productor; políticas; pobreza; regulación.
Abstract: An overview of the state of the rice market in Costa Rica between 2002-2013 is described in the article, characterizing the agro-chain that encompasses the market from production to marketing. In addition, relevant indicators are included to determine the country's position regarding food security, based on the data provided by Costa Rica's National Rice Corporation (CONARROZ). Finally, the article describes the impact of the rice market on the socioeconomic sector and the efforts made by the State in terms of public policies in the development of this sector.

Keywords: food security; producer; policies; poverty; regulation.

1 Master en Economía del Desarrollo. Se desempeña como docente e investigadora de la Escuela de Economía de la Universidad Nacional, Costa Rica. Correo electrónico: yendry.sanchez.picado@una.cr.

2 Master en Economía del Desarrollo, docente e investigadora de la Escuela de Economía de la Universidad Nacional, Costa Rica.rmaria.vega.solano@una.cr. 


\section{Introducción}

Para el desarrollo de la investigación, plantea como objetivo general analizar el estado actual del mercado del arroz en Costa Rica durante el periodo 2000-2013, tomando en consideración la valoración de los actores, sus interrelaciones en la cadena del arroz y algunos indicadores de producción del sector y su afectación a través del tiempo.

Se desarrolla el coeficiente de Autosuficiencia y Dependencia Alimentaria para así, vislumbrar la posición del país en materia de seguridad alimentaria. Posteriormente, se estudia el impacto económico y social del mercado del arroz sobre el desarrollo económico del país; además, se describen las leyes y políticas públicas implementadas por el Estado para mejorar la situación del mercado del arroz nacional en los últimos años; todo este estudio se realiza a partir de los datos suministrados por la Corporación Nacional Arrocera (CONARROZ), así como de la información recopilada por medio de cuestionarios estructurados que se aplicaron a los miembros de esa entidad durante el Congreso Arrocero Nacional en el año 2014.

\section{Metodología}

Con el fin de responder al objetivo planteado se realizó un análisis mixto; es decir, se recolectaron y analizaron datos proporcionados por CONARROZ y brindados por los 82 miembros de la corporación que fueron encuestados; esta información fue tanto cuantitativa como cualitativa y permitió obtener resultados concretos y veraces sobre el tema. De manera más desagregada, el enfoque cualitativo se basó en estudios de percepción objetivista que muestra la realidad mediante el análisis de hechos históricos y actuales, permitiendo que la investigación desarrollada alcanzara a ser un estudio explicativo a la situación del país.

Por su parte, el enfoque cuantitativo se dio bajo la modalidad descriptiva mediante la recolección de datos y estadísticas ${ }^{3}$. Así mismo, se utilizó información secundaria dada por instituciones como el Ministerio de Economía y Comercio (MEIC), Banco Central de Costa Rica (BCCR), Ministerio de Planificación y Política Económica (MIDEPLAN) entre otros, permitiendo dar un abordaje más amplio del mercado arrocero costarricense. A partir de la recolección de datos, se calcularon coeficientes e índices como el ratio de concentración y el Índice de Herfindahl-Hirschman (IHH).

\section{La cadena del arroz en Costa Rica}

El mercado del arroz en Costa Rica cuenta con una cadena de seis etapas, en las que se interrelacionan los actores del sector: producción e importación de insumos, producción de arroz granza, industrialización (agroindustrias), comercialización y distribución, comercio exterior y consumo final, a lo que se agrega las actividades de apoyo (insumos y servicios) y 
el ambiente institucional (leyes, normas, políticas). La Figura 1 muestra de manera resumida la cadena institucional del sector arrocero.

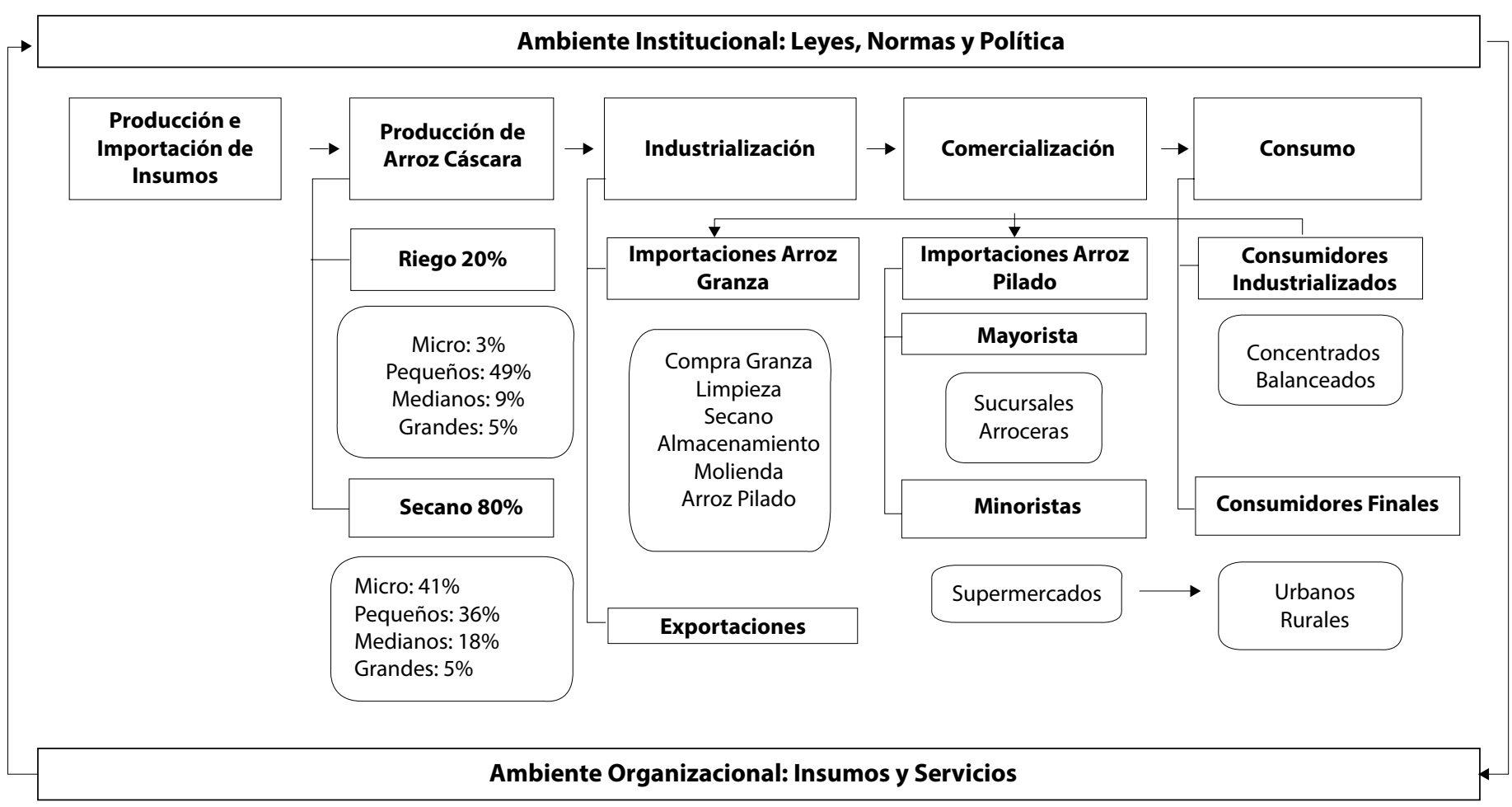

Figura 1: Costa Rica: Segmentos de la cadena arrocera costarricense. Fuente: elaboración propia. 
La actividad arrocera inicia con la preparación de los terrenos y la aplicación de algunos insumos que garanticen un ambiente adecuado para el desarrollo de la semilla. Posteriormente, se procede con la siembra propiamente para obtener la granza de arroz o arroz con cáscara. Una vez listo es transportado a las agroindustrias para venderlo, seguidamente los industriales se encargan del secado, almacenamiento y molienda del arroz en granza hasta obtener el arroz pilado.

Las agroindustrias también obtiene el producto a través de la importación de arroz granza, que llega seco y limpio para almacenar y seguir su proceso dentro de la cadena. Los agroindustriales o los importadores adquieren el arroz pilado, el cual luego es obtenido por los mayoristas y posteriormente por los minoristas, hasta que finalmente llega a manos de los consumidores.

En Costa Rica la producción del arroz es realizada durante dos periodos en el año, siendo la primera siembra la que mayor cobertura de producción genera; por ejemplo, para el período 2000-2013 en promedio 67\% de la cosecha total se produjo en la primera siembra, esto de acuerdo con los datos aportados por CONARROZ; en el país estos periodos varían según la región de cultivo.

La producción de este grano básico es generada mayoritariamente por productores denominados micro/pequeño (79\%), mientras que el $16 \%$ y el $5 \%$ corresponde a medianos y grandes productores respectivamente, en donde el método secano es el más empleado ya que cerca del $78 \%$ de ellos lo utilizan, mientras que el método de riego solo representa aproximadamente el $22 \%{ }^{4}$.

La producción de arroz en el país durante el 2000-2013 presenta un comportamiento inestable, siendo el periodo 2006-2007 el de menor capacidad productiva con 171 117,49 toneladas métricas mientras que durante el periodo $2010-2011$ se presentó la mayor productividad con 290474,70 toneladas métricas, situación que es evidente al estudiar el comportamiento de la tasa de crecimiento interanual de la producción, ya que su tendencia mostró un comportamiento fluctuante (Figura 2), teniendo como principales hallazgos la notable propensión al alza del 20082009 , período en el cual se da la mayor tasa de crecimiento $(27,16 \%)$, respaldado básicamente por la correlación positiva que esta mantiene con el área sembrada y cosechada (durante el 2007-2008, el área sembrada y cosechada fue de 54 042,66 ha, mientras que para el 2008-2009 esta fue de 63 329,09).

En cuanto a los períodos 2001-2002, 2005-2006 y 2012-2013 se registraron las menores tasas de crecimiento interanuales, que incluso llegaron a ser negativas $(-19,34 \%,-13.93 \%$ y $-22,99 \%$ respectivamente), debido a factores climáticos que disminuyeron el área sembrada ubicándola

$4 \quad$ La siembra de arroz a través del riego consiste en que el agua que requiere el cultivo es provista por el hombre en cualquier momento, mientras que en el secano el agua que requiere el cultivo únicamente proviene de las lluvias. 
para el 2000-2001 en 67 015,35 ha, mientras que para 2001-2002 fue de 56 326,20 ha., asimismo, se pasó de cosechar en el 2004-2005, 60 414,04 ha. a 54 092,82 ha. en el 2005-2006.

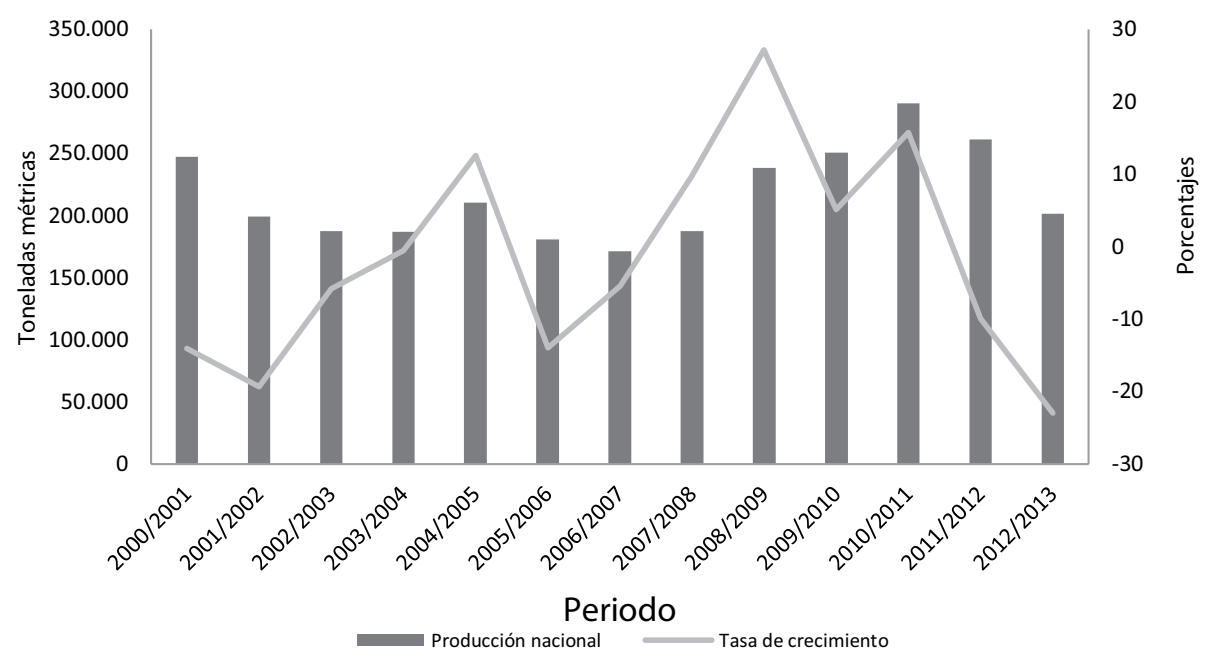

Figura 2. Costa Rica: Producción de arroz granza limpia y seca Según: Tasa de crecimiento Interanual En: Toneladas métricas 2000 - 2013. Fuente: Elaboración propia con base en datos de Inteligencia de Mercados, CONARROZ.

Al igual que el comportamiento mostrado por la producción, en la Figura 2 es posible constatar que para el período 2006-2007 el área sembrada se caracterizó por tener tasas de decrecimiento de hasta 12,64\% respecto al año anterior; dichas reducciones obedecieron a factores climáticos adversos de la época, problemas de manejo agronómico y a la disponibilidad de variedades de semillas de acuerdo a la zona productiva. 


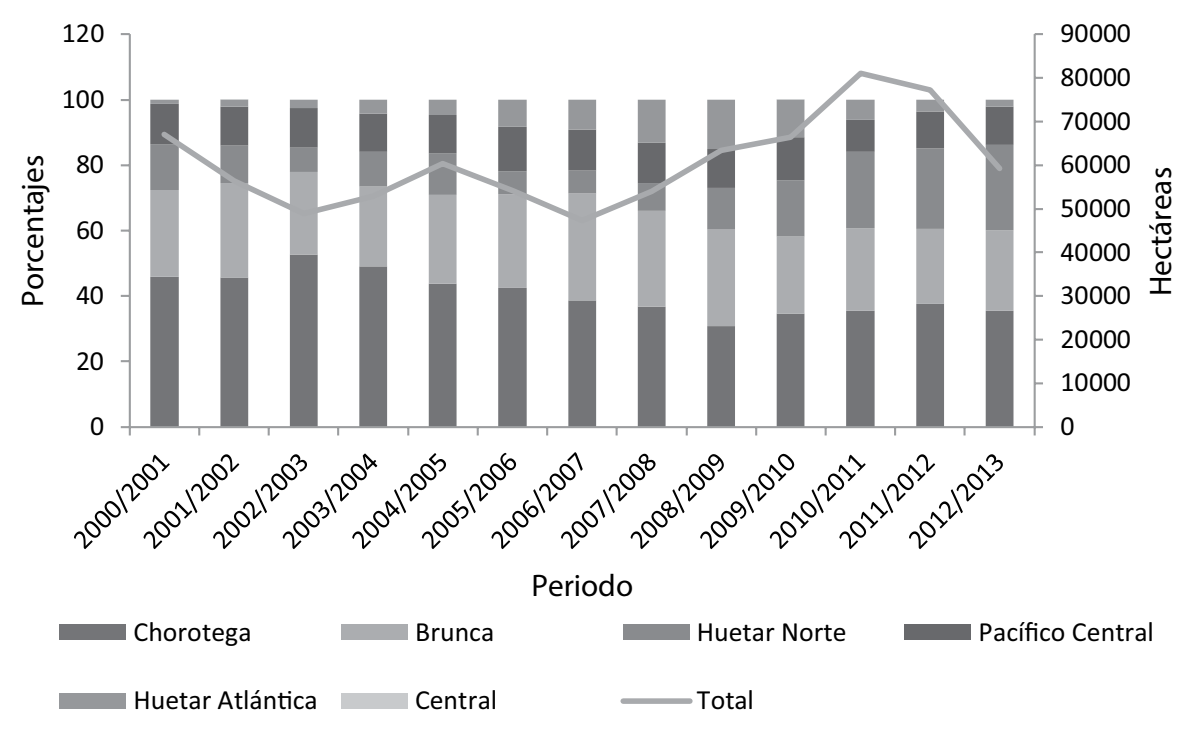

Figura 3. Costa Rica: Área sembrada. En: Participación Relativa y Hectáreas. Según: Región. 2000 - 2012. Fuente: Elaboración propia con base en datos de Inteligencia de Mercados, CONARROZ.

Es importante mencionar que la cantidad de áreas sembradas varía de acuerdo a la región, siendo la Chorotega y la Brunca las que poseen mayor cobertura de hectáreas sembradas con $35 \%$ y $24 \%$ respectivamente para el periodo 2012-2013; situación contraria al Pacífico Central que siembra $11 \%$ y la Huetar Atlántica con tan solo $2 \%$ de la producción total de acuerdo con datos de CONARROZ.

La cantidad de agroindustrias es otro determinante de la producción de arroz en Costa Rica pues, mientras más oferta de plantas industrializadoras mayor es el incentivo para los productores de cosechar arroz, principalmente si estas plantas se encuentran cerca del área sembrada, ya que son los agricultores de arroz quienes se tienen que encargar de llevar su cosecha hasta las agroindustrias.

La mayor cantidad de agroindustrias están ubicadas en la región Chorotega, principal región con mayor área sembrada y mayor cobertura de las compras con alrededor del 52,56\%; no obstante, en el periodo de estudio, las agroindustrias de dicha región han tendido a disminuir, pasando de tener once agroindustrias en el periodo 2000-2001 a tan solo ser seis en el periodo 2012-2013. 
Otro aspecto relevante a evaluar es el comportamiento del sector externo, la producción y el consumo mundial (local e internacional), para así analizar los flujos de importaciones y exportaciones nacionales e internacionales y por ende, la importancia del comercio en el mercado arrocero; en el periodo de estudio (2000-2013) a nivel internacional hubo flujos de comercio, producto de la diferencia entre la producción y el consumo de arroz, donde a nivel mundial la producción supera el consumo. En cuanto a Costa Rica durante el periodo 20012007 y en el año 2012, el consumo fue mayor a la producción; situación contraria a los periodos 2008-2011, debido a la existencia de sobreproducción local por aumentos en el área sembrada y a la absorción de trabajadores del sector construcción, lo que hizo disminuir el flujo de importaciones.

Con datos de la Organización de las Naciones Unidas para la Alimentación y la Agricultura (FAO), el promedio de importaciones mundiales para el periodo en estudio fue de 28570,85 mil toneladas, mientras que para Costa Rica esta cifra se ubicó en 88,85 mil toneladas.

Durante el período 2006-2013 las importaciones crecieron a una tasa promedio del 28\%; sin embargo, es importante mencionar que para el 2011-2012 se da un crecimiento anormal del 244\% (las importaciones pasaron de 33 588,28 TM del 2009-2010 a 115 420,90 TM para el siguiente período), lo que contrasta con la tendencia de decrecimiento en las importaciones durante la mayoría del período en referencia.

Para el 2010-2011 las importaciones disminuyeron hasta ubicarse en 33 558,28 T.M., debido principalmente a la reducción acelerada de arroz en cáscara producto del incremento de la producción nacional. Siendo ésta la que cubrió el $69 \%$ de la demanda total y el $31 \%$ restante se resguardó con importaciones, ello muestra una dependencia en ese mismo porcentaje, valor mucho menor al registrado durante el 2007-2008 que fue de 50\%. Para el 2010-2011 la dependencia fue de tan solo 10\%, producto de la sobreproducción nacional del 2009-2010.

Según el Informe Estadístico de CONARROZ 2011-2012, en este periodo no fue necesario realizar importaciones por desabasto ${ }^{5}$, ya que el faltante de arroz fue cubierto por las importaciones por contingente ${ }^{6}$ CAFTA-RD $^{7}$ y otras importaciones realizadas por arroceras o terceros; de las cuáles 100143 toneladas fueron de arroz en granza, 11361 toneladas fueron de arroz pilado y las restantes 3872 correspondieron a otros tipos de arroz (Figura 4).

5 Importación que se da por insuficiencia de la producción local para cubrir el consumo nacional.

6 Término que hace referencia a la cantidad de una mercancía que como máximo se permite importar en un país durante un determinado periodo de tiempo.

7 Tratado de Libre Comercio para la República Dominicana y Centro América (CAFTA), que entro en vigencia en el 2009. 


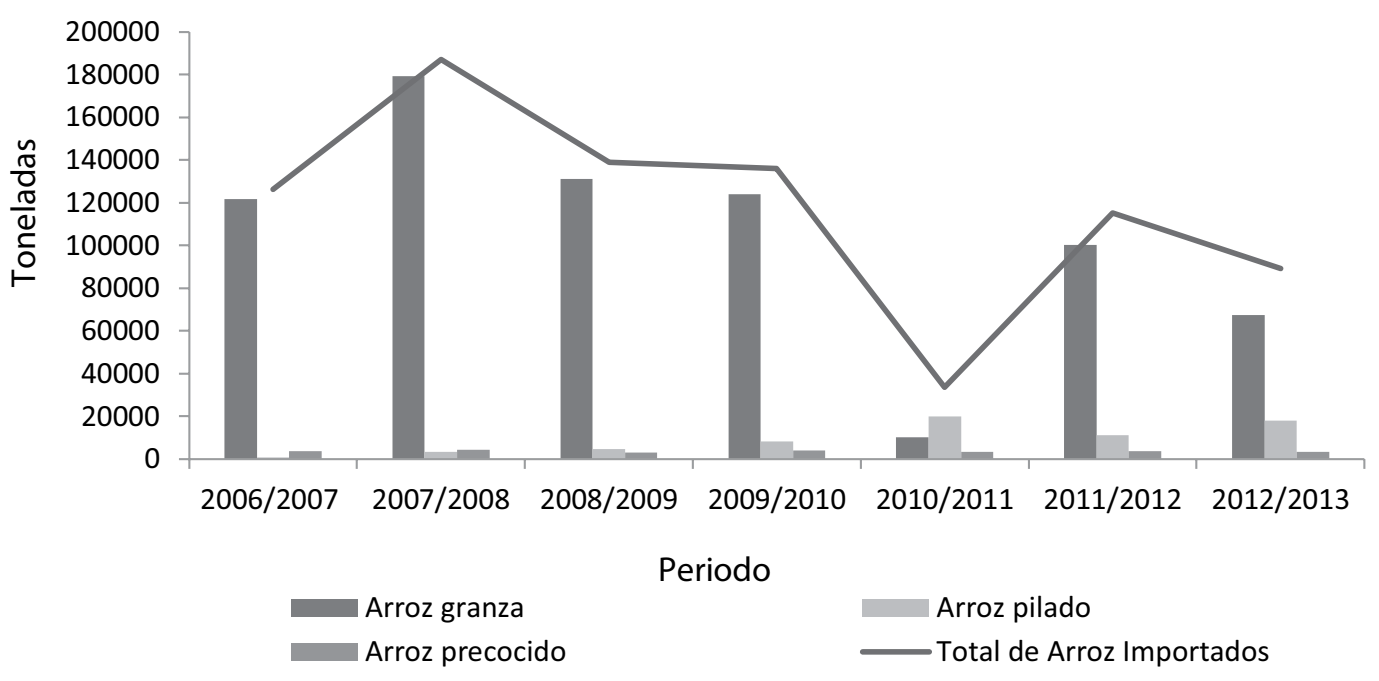

Figura 4. Costa Rica: Importación total de arroz. Según: tipo de arroz. En: toneladas. 20062013. Fuente: Elaboración propia con base en datos suministrados por Inteligencia de Mercados, CONARROZ.

Para el periodo 2000-2013 las exportaciones ${ }^{8}$ totales de arroz fueron en promedio de 4914 TM, en donde se puede confirmar que durante los lapsos 2001-2002 y 2007-2008 presentó la mayor exportación de arroz del país y se vendió más de 10000 TM, lo cual pudo ser resultado de la inestabilidad económica producto del impacto en la economía mundial por los atentados del 11 de septiembre del 2001, así como el inicio de los problemas y consecuente quiebra de Lehmman Brothers en 2008 que ocasionaría la crisis económica mundial al año siguiente (2009).

Se observa que después de estos picos de exportación de arroz (Figura 5), se da un proceso de dos a tres períodos de tiempo con descenso en las exportaciones, para luego retomar su crecimiento, que durante este periodo de estudio ha estado situado alrededor de las 5000 TM.

8 Compuestas por una serie de partidas arancelarias clasificando los usos del arroz exportado como por ejemplo: las partidas 1006101000 para siembra, la 1006109090 granza los demás, 1006200000 descascarillado y 1904901000 precocido, entre otras. 


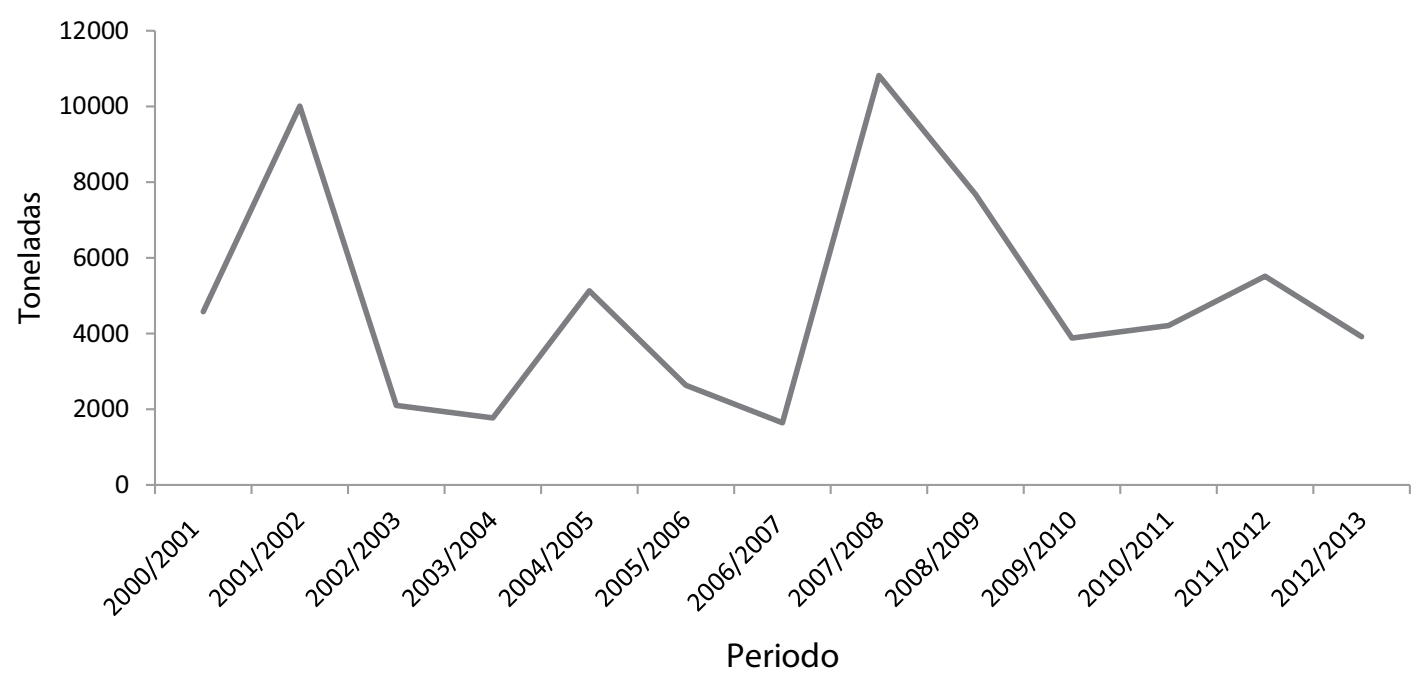

Figura 5. Costa Rica: Evolución de las exportaciones totales de arroz. En toneladas. 20002013. Fuente: elaboración propia con base en datos suministrados por Inteligencia de Mercados, CONARROZ.

Por último y no menos importante, se encuentra el eslabón de la cadena del arroz denominado consumo, el cual mantuvo una tendencia muy regular a lo largo del período 2000-2013 de acuerdo con los datos suministrados por Inteligencia de Mercados de CONARROZ, con un promedio de consumo per cápita de 51,78 kilogramos de arroz pilado; no obstante, este indicador mostró algunas variaciones significativas, entre ellas se encuentran una reducción del período 2007-2008 hasta el 2010-2011, esto debido principalmente al contexto mundial de crisis alimentaria (la cual ocasionó un incremento en el precio de los alimentos, e incluso, según estimaciones del Banco Mundial, FAO y el Fondo Monetario Internacional (FMI) los precios de los alimentos no bajarían antes del 2015); sin embargo, al finalizar el período 2012 el consumo se recuperó y alcanzó un total de 53,71 kg/per cápita,1,63 p.p. por encima del promedio del período.

Aunado a lo anterior y dado que el arroz es considerado un producto estratégico para la seguridad alimentaria y nutricional de la población, el Estado en los últimos años ha apoyado la actividad arrocera, mediante la Ley de Creación de la Corporación Arrocera Nacional № 8285; asimismo, el país cuenta con un marco regulatorio desde 1995 que busca mitigar las malas prácticas de los mercados a partir de la Ley de Promoción de la Competencia y Defensa Efectiva del Consumidor No 7472. 


\section{Mercado del arroz: una mirada a la seguridad alimentaria}

Existen dos formas para estimar la seguridad alimentaria: la autosuficiencia alimentaria y la autonomía alimentaria; ambos indicadores aseguran que las personas poseen un adecuado nivel de vida, en donde es posible el acceso a alimentos tanto física como económicamente.

El indicador para la autosuficiencia alimentaria, prioriza la satisfacción de necesidades alimentarias de la población local con suministros internos, reduciendo de tal forma la dependencia del comercio (soberanía alimentaria); y evitando así los vaivenes y fluctuaciones de las economías externas.

En cuanto a la autonomía alimentaria, esta considera las posibilidades de la realización del comercio internacional, ya que consiste en generar un cierto nivel de producción interna de alimentos y generar además, la capacidad para importar cuando sea necesario.

De acuerdo con lo anterior, se hace importante conocer el comportamiento que ha tenido el país en la producción de alimentos para cubrir el consumo interno del arroz para el periodo 20002013. Debido a lo anterior, a continuación, se procederá con el cálculo de estos dos coeficientes (Autosuficiencia y dependencia alimentaria).

En relación con el coeficiente de autosuficiencia ${ }^{9}$ del arroz para el periodo 2000-2013, a pesar de mostrar porcentajes entre $50 \%$ y $82 \%$, no revela una tendencia estable, tal como lo evidencia la siguiente figura.

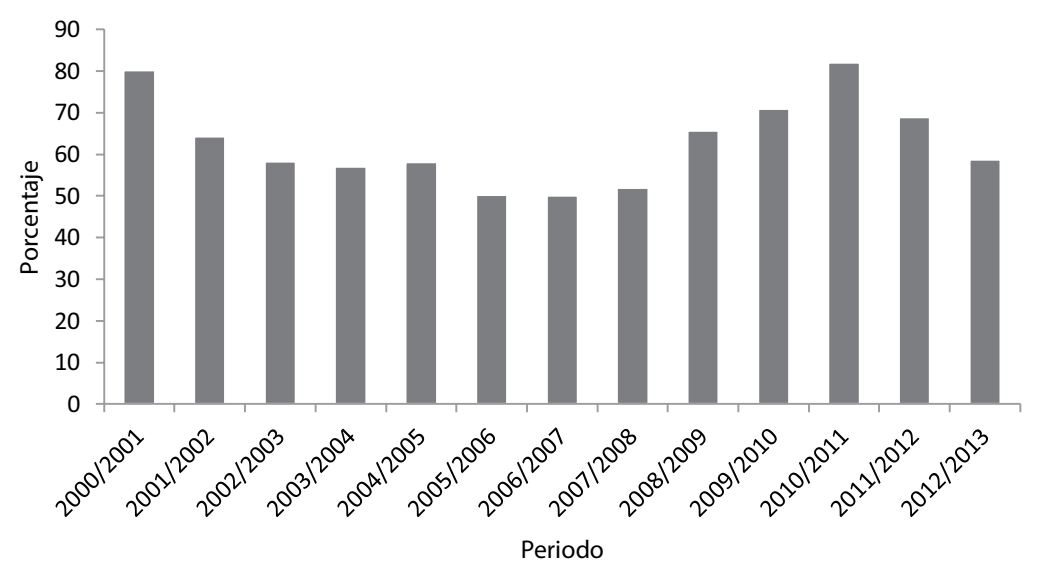

Figura 6. Costa Rica: Coeficiente de autosuficiencia. En: porcentajes. 2000-2013. Fuente: Elaboración propia con base en datos suministrados por Inteligencia de Mercados, CONARROZ.

9 Consumo cubierto por producción nacional. 
La inestabilidad del coeficiente de autosuficiencia está explicada principalmente por la existencia de periodos como el 2005-2006 y 2006-2007, en que el mismo se ubicó en 50,28\% y $50,07 \%$ respectivamente de acuerdo con los datos de Inteligencia de Mercado de CONARROZ; lo cual conllevó a que el consumo se solventará por las importaciones (49\% aproximadamente) debido a la disminución de las áreas sembradas, fenómenos climáticos, reducciones del crédito al sector, entre otros.

No obstante, desde el periodo 2007 hasta el 2011, bajo una coyuntura internacional de crisis alimentaria ${ }^{10}$, el coeficiente de autosuficiencia en Costa Rica presentó año tras año variaciones positivas hasta alcanzar en el 2011 un coeficiente del 82\%, es decir, ante el panorama mundial de desabastecimiento de productos agrícolas, la producción nacional arrocera realizó esfuerzos para que la población no careciera de este producto.

En cuanto al coeficiente de dependencia alimentaria del arroz para el periodo 2000-2013, este muestra un comportamiento contrario a la producción, ya que incrementa conforme la producción arrocera disminuye.

Dichocoeficienterefuta la situación mostradaanteriormente con el coeficiente deautosuficiencia, puesto que para el 2007-2008 la dependencia alimentaria era del 50,45\%, mientras que para los siguientes periodos de crisis alimentaria mundial, la producción nacional de arroz alcanzó mayores niveles, logrando de tal forma que la dependencia de las importaciones fuera solo del 10,28\% para el periodo $2010-2011$.

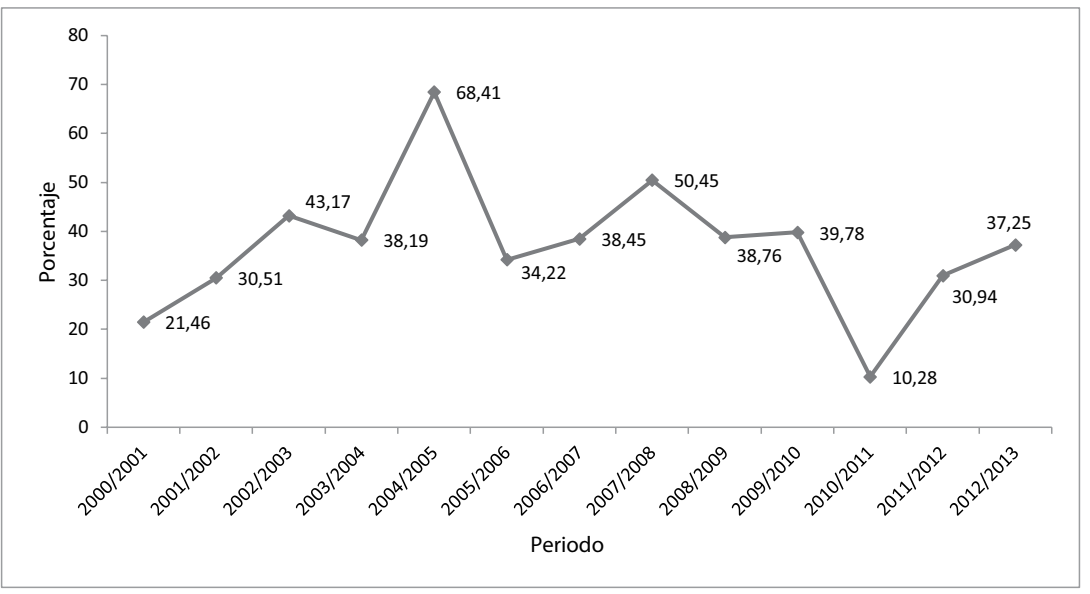

Figura 7. Costa Rica: Dependencia alimentaria. En porcentajes. 2000-2013. Fuente: Elaboración propia con base en datos suministrados por Inteligencia de Mercados, CONARROZ. 
Cabe señalarquelaaperturadelcomercioagropecuario delos países menos desarrollados provoca que en los momentos de auge internacional puedan elevar los ingresos considerablemente, siendo una fuente importante de divisas actuando como catalizador para el desarrollo global; no obstante, en los momentos de crisis puede ser una amenaza para el consumo de la población de los países dependientes de las importaciones (Faletto, 1977), puesto que los productores de los países exportadores netos procurarán principalmente el abastecimiento nacional que solventar las necesidades de sus socios comerciales.

Si bien, para la mayoría de los países las importaciones de alimentos son ya una fuente importante de suministros y seguirán contribuyendo a la seguridad alimentaria; la dependencia del comercio puede presentar riesgos como el desabastecimiento, la especulación, la incertidumbre y las fluctuaciones en los precios del mercado mundial.

\section{Efectos socioeconómicos del mercado del arroz en Costa Rica}

La globalización, la apertura de los mercados y la formación de bloques, tratados y acuerdos comerciales, han sido las transformaciones recientes de las relaciones económicas a nivel mundial. Todo esto ha llevado en las últimas décadas a los países latinoamericanos a establecer reformas y políticas que proporcionen seguridad y estabilidad a los precios de los mercados e ingresos de los productos en general y básicos en particular.

No obstante, la liberalización de precios es dada por políticas comerciales que buscan bajar el precio de bienes y servicios mediante el estímulo de la producción industrial y de esta forma aumentar la oferta, pero en la práctica los efectos que pueden tener dichas políticas van más allá de una rebaja en el precio de un producto o de un aumento de la oferta. Por ejemplo ${ }^{11}$, según estudios de Combbes (2002), la liberalización del comercio en países en desarrollo los hace más vulnerables a los shocks externos, lo cual tiene un efecto adverso en la estabilidad de los términos de intercambio ${ }^{12}$.

A nivel de Centroamérica, en el año 2010 los productores de granos básicos aumentaron, debido a avances de la frontera agrícola o a reformas agrarias. Sin embargo, en muchos casos las condiciones de esta ampliación han sido a partir de condiciones precarias, sin acceso a crédito o tecnologías que permitan la mejora en el rendimiento. Por su parte, en Costa Rica se generó una reducción en la producción interna, asociada en cierta parte a una reconversión productiva de los hogares hacia cultivos como la palma africana, la piña y el café, por lo que dicha disminución fue compensada con importación.

\footnotetext{
11 Tomado de Eco-finanzas: http://www.eco-finanzas.com/diccionario/T/TERMINOS_DE_INTERCAMBIO.htm.

12 Relación que existe entre los precios medios de importación y de exportación, para todos los bienes y servicios.
} 
Pero ante dicha situación ¿Cuál es la estado socioeconómico en el que se encuentran los productores arroceros en el país?, como se mencionó anteriormente, este sector está compuesto principalmente por pequeños productores con un ratio de concentración promedio de 0,79 seguido por medianos y grandes productores con un promedio de 0,15 y 0,05 respectivamente para el periodo 2000-2013, ubicados mayoritariamente en la regiones Chorotega y Brunca con una participación del 35\% y 24\% para el 2012-2013.

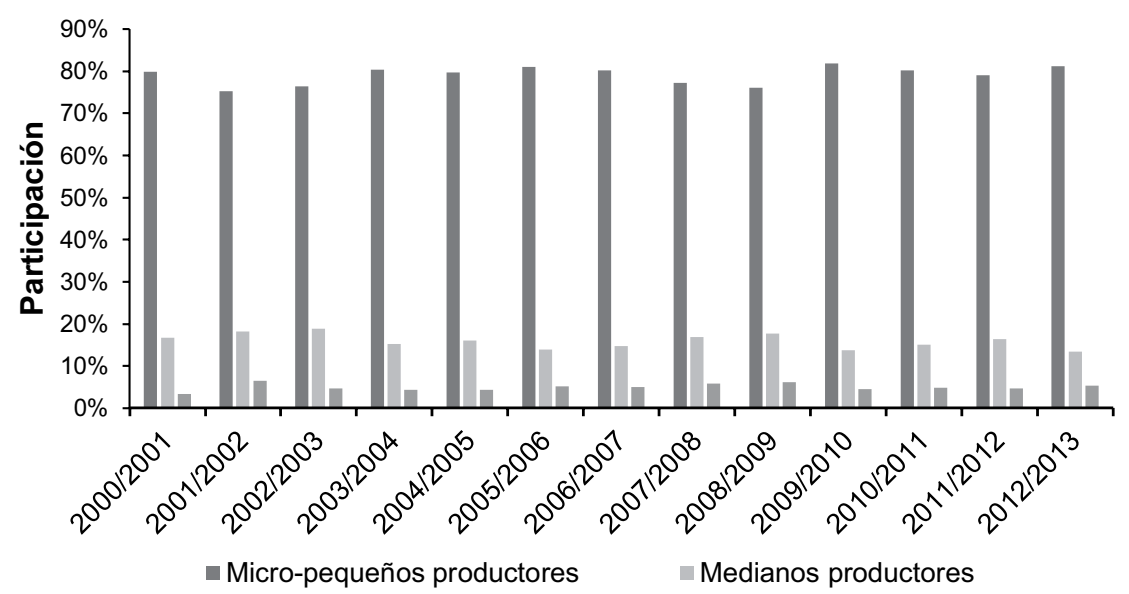

Figura 8. Costa Rica: productores de arroz. Según: Índice de Concentración (C1). Por: estratos. 2000-2013 Fuente: elaboración propia con base en datos de Inteligencia de Mercados, CONARROZ.

Misma situación es corroborada por cálculo del índice de concentración Herfihdhl - Hirschman, el cual si alcanza un valor cercano a 10000 , indica que la industria arrocera en productores es altamente concentrada, por el contrario si este valor tiende a cero no hay concentración en dichos estratos.

Este índice muestra una tendencia al alza en la concentración del número de productores; entre los años 2000-2013 el promedio del índice se encontró en 6 544,26 puntos, siendo el 20012002 el periodo con menor concentración de 6 046,55 puntos, mientras que los años 20092010 la concentración alcanzó valores de 6897 aproximadamente, lo cual evidencia un nivel importante de concentración, tal y como se ilustra en la figura siguiente: 


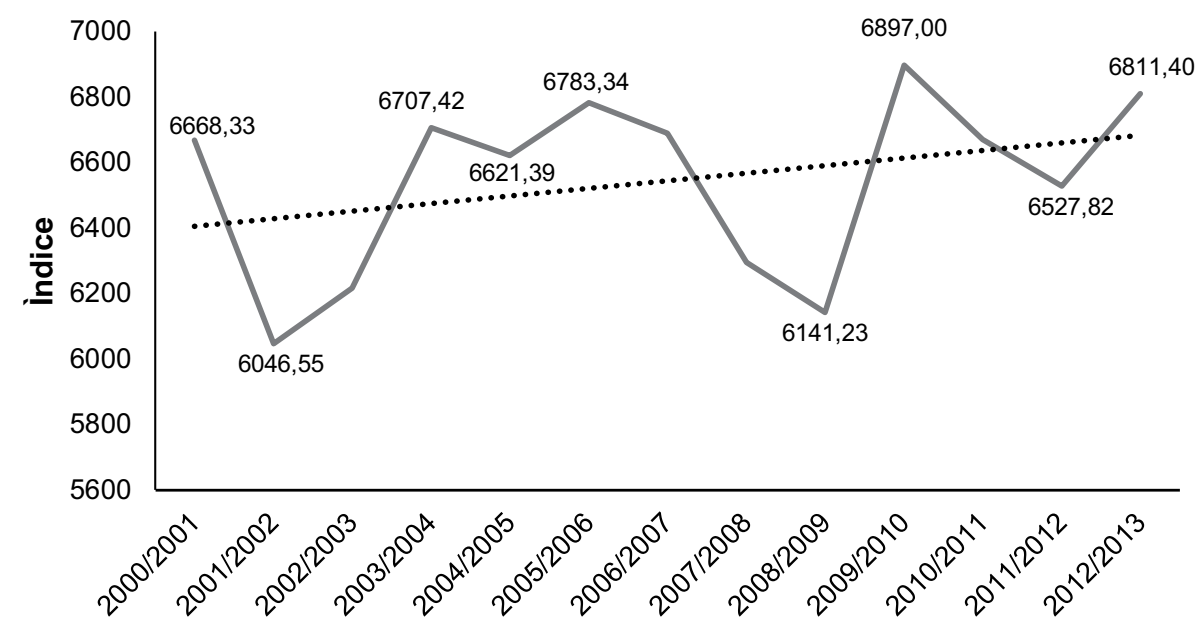

Figura 9. Costa Rica:Total de productores de arroz. Según: Índice Herfindahl - Hirschman. 2000-2013. Fuente: Elaboración propia con base en datos de Inteligencia de Mercados, CONARROZ.

Por tanto, ante dicha situación y analizando indicadores socioeconómicos del país tales como desempleo, pobreza, desigualdad, se puede vislumbrar la susceptibilidad en la que se encuentran distintas regiones del país con respecto al desarrollo del sector arrocero.

El desempleo en Costa Rica es elevado en las principales áreas productoras de arroz durante el 2010-2013 ya que, mientras el promedio nacional de desempleo para ese periodo era de 7,81\% de la fuerza de trabajo, regiones como la Chorotega y Huetar Atlántico reportaron tasas de $10,28 \%$ y $9,29 \%$ respectivamente, afectando tanto a hombres como a mujeres.

En cuanto a la situación de pobreza en la que se encuentran dichas regiones, según datos estimados, las tres principales áreas productoras del grano poseen tasas promedio de pobreza, pobreza no extrema y pobreza extrema, superiores a las nacionales, donde resalta la situación de la región Brunca, en la que del 2010 al 2013 la pobreza extrema ha aumentado aproximadamente 1,5 puntos porcentuales; sin embargo, se ha reducido la pobreza no extrema en cerca de1,1 punto porcentual ( $22,3 \%$ en 2010 y $21,2 \%$ en 2013$)$, en donde cabe mencionar que la principal fuente de empleos en la zona se asocia al sector primario.

El panorama de la región Chorotega sigue el comportamiento mostrado por la región Brunca, ya que los porcentajes de pobreza han venido en aumento; pasó de tener un porcentaje de pobreza no extrema de $21,4 \%$ en 2010 a $21,6 \%$ en 2013 . Por su parte, la pobreza extrema aumentó alrededor de 1,4 puntos porcentuales, ubicándose en 12,6\% en el 2013. 
Tabla 1.

Costa Rica: regiones productoras de arroz. Según condición de pobreza. En porcentaje promedio, 2010-2013.

\begin{tabular}{lccc}
\hline \multicolumn{1}{c}{ Región } & Pobreza & Pobreza no extrema & Pobreza Extrema \\
\hline Chorotega & 33,2 & 21,2 & 12,0 \\
Huetar Norte & 25,6 & 16,7 & 8,9 \\
Huetar Atlántica & 27,6 & 18,4 & 9,2 \\
Brunca & 34,4 & 21,1 & 13,3 \\
Promedio Nacional & 21,1 & 14,8 & 6,3 \\
\hline
\end{tabular}

Fuente: Elaboración propia con base en datos del INEC.

La pobreza y el desempleo no afectan únicamente a los productores de arroz, sino también a quienes lo consumen; en términos generales, según el Índice de Precios al Consumidor (IPC) el rubro de alimentos y bebidas, donde se incluye el arroz tiene un peso relativo de $18,61 \%$, siendo el de mayor peso, seguido únicamente por el rubro de transportes; esto demuestra su afectación en el gasto de los costarricenses.

Específicamente, el arroz ha presentado una variación anual promedio de los precios de $63 \%$ entre el periodo 2006-2013, cambio menor al sufrido por productos como los derivados del maíz y el trigo, los frijoles, las pastas y los huevos, en el mismo periodo de estudio. Es importante resaltar que en Costa Rica, el arroz el único producto de la canasta básica con precio regulado.

Más específicamente, cuando se analiza el incremento del precio de forma mensual tal y como se muestra en la siguiente figura, el arroz presenta menor variación en comparación con otros artículos de la canasta básica, como las tortillas de maíz y los frijoles.

Este aspecto es sumamente importante ya que inhibe un aumento excesivo en el precio de dicho producto y por ende permite a las personas con mayor vulnerabilidad por su condición socioeconómica seguir accediendo a este bien de primera necesidad en la mayoría de hogares costarricenses; cabe mencionar que a partir del decreto del 2013 que fijó el precio del arroz de una mayor cantidad de calidades el índice ha tendido a la baja y a disminuir la variación. 


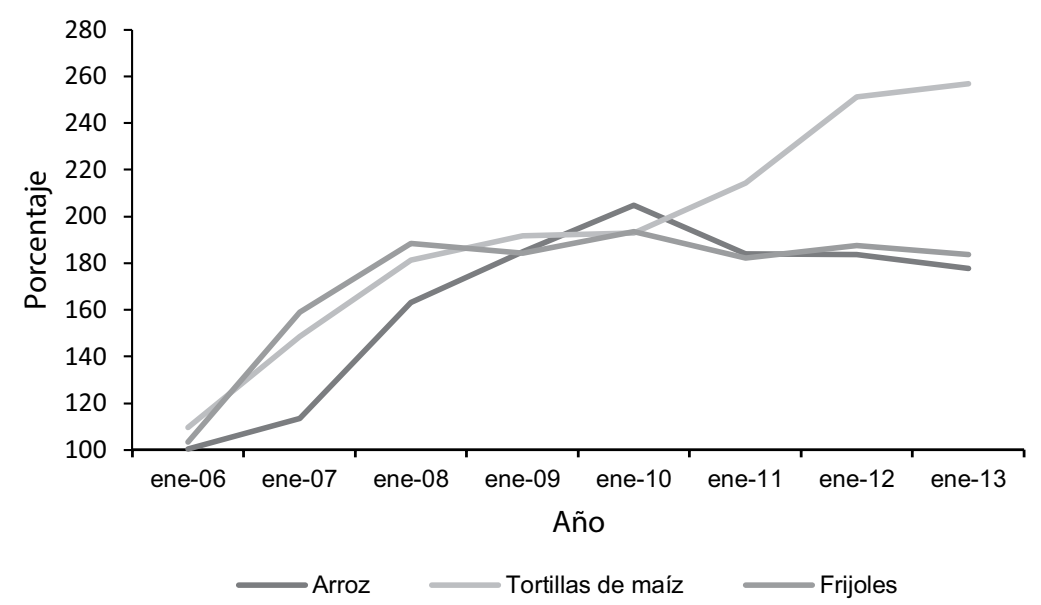

Figura 10. Costa Rica: Productos de la Canasta Básica. Según: Variación mensual. En: Porcentaje. 2006-2013. Fuente: Elaboración propia con base en datos del INEC.

En Costa Rica el arroz es consumido por casi la totalidad de la población dado su alto valor nutricional; si se analiza la composición de la población por quintiles ${ }^{13}$ según la Encuesta Nacional de Ingresos y Gastos (ENIGH) 2013, los ciudadanos pertenecientes al primer quintil destinan de su ingreso cerca de $41,3 \%$ al consumo de alimentos y bebidas, siendo el grupo de pan y cereales el más importante, con $23 \%$. Mientras que el quinto quintil gasta únicamente el $21,1 \%$ en alimentos y bebidas, de los cuales $8,8 \%$ corresponde a comidas y bebidas fuera del hogar.

Por otra parte, respecto a la distribución del ingreso determinada por el coeficiente de Gini, este indicador muestra una situación poco alentadora para el país, pues ha aumentado cerca de 4 puntos, lo que evidencia un deterioro en la distribución del ingreso, tal y como se presenta en la figura 10, donde a nivel de regiones, las que muestran mayor desigualdad son la Chorotega $(0,532)$ y la Brunca $(0,525)$, situación que puede repercutir sobre los niveles de pobreza nacionales y regionales.

13 Un quintil corresponde a la quinta parte de una población estadística ordenada de menor a mayor. 


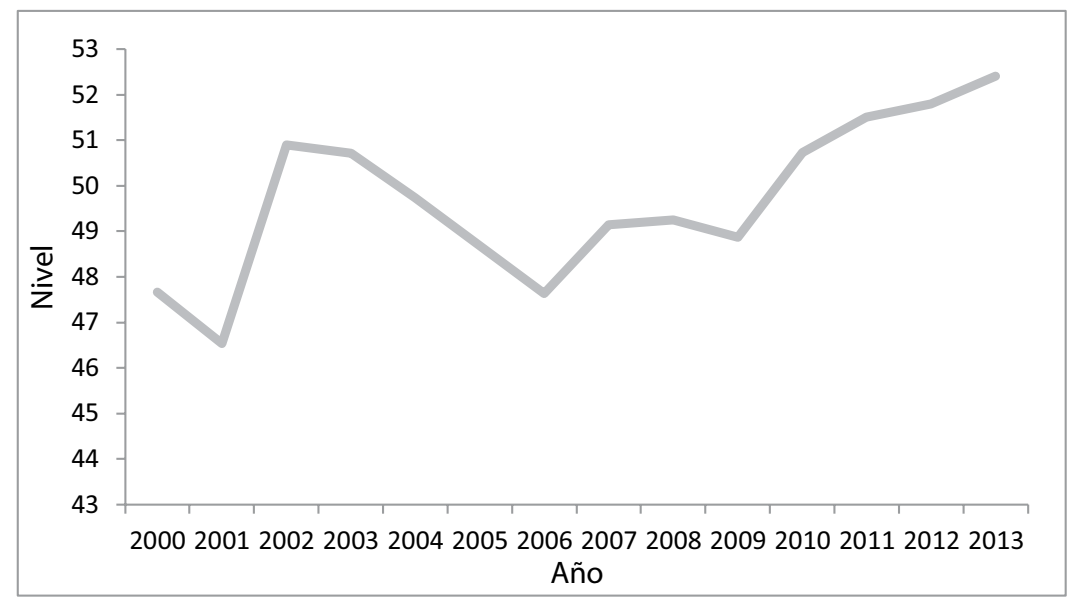

Figura 11. Costa Rica: Coeficiente de Gini. En: Niveles. 2000-2013. Fuente: Elaboración propia con base en datos del Banco Mundial.

Por tanto, la disminución de la producción nacional, el aumento del desempleo y los altos niveles de pobreza en las regiones productoras de arroz llaman la atención sobre la necesidad de tomar medidas que lleven a la protección de los sectores más vulnerables y de la población en su conjunto, que les permita el acceso al empleo y a los alimentos básicos como el arroz.

Recordando que las principales zonas productoras de arroz en el país presentan elevadas tasas de desempleo y de pobreza (no extrema y extrema), planteando al Estado la necesidad de crear políticas que fortalezcan la seguridad alimentaria de las zonas productoras y así, evitar repercutir negativamente sobre la agricultura familiar y los consumidores nacionales.

\section{Políticas públicas en el mercado del arroz}

Las diferentes coyunturas mundiales han hecho que el Estado costarricense se encuentre realizando esfuerzos constantes en la búsqueda de mercados con niveles adecuados de competitividad, en donde tanto productores como consumidores no degeneren prácticas que limiten la libre competencia; para dar respuesta a este esquema se han establecido leyes, reformas y políticas que permitan ciertas regulaciones de los mercados.

Este proceso da inicio en Costa Rica en la década de los cincuenta, siendo la Ley de Defensa Económica (Ley $\mathrm{N}^{\circ} 1208$ ) creada en octubre de 1950 la primera reforma que busca regular el comercio y así, poder garantizar a los consumidores precios de equilibrio sin alzas abusivas. El principal objetivo de la mencionada ley era velar por los derechos y las obligaciones de los participantes en la relación comercial. 
Posteriormente, se creó la Ley de Protección al Consumidor (Ley N 5665), en febrero de 1975, con el fin de dar mayor control a las especulaciones y al comportamiento de los precios, debido principalmente a los fenómenos inflacionarios que atravesaba el país para finales de los años setenta ${ }^{14}$, siendo el compromiso, alinear el mercado ante alzas de precios bajo un sistema de ajuste de ganancias entre participantes.

Por otra parte, en enero de 1995 se creó la Ley de Promoción de la Competencia y Defensa Efectiva del Consumidor (Ley $\mathrm{N}^{\circ}$ 7472), vigente a la fecha, que por medio del artículo 5 determina los casos en donde procede la regulación de precios: "...Para el caso específico de condiciones monopolísticas y oligopólicas de bienes y servicios, la Administración Pública regulará la fijación de los precios mientras se mantengan esas condiciones." (Ley № 7472, Art 5, pág. 4).

Es decir, la ley $\mathrm{N}^{\circ} 7472$ procede en aquellas estructuras del mercado en donde una empresa (monopolio) o pocas empresas (oligopolio) participan en la producción de un bien y este no tiene sustitutos cercanos, por lo que son capaces de influir en los precios del mercado.

En Costa Rica, el mercado del arroz ha sido unos de los productos de la canasta básica que desde finales de los años 1980 viene enfrentando cambios, ya que inicialmente era liderado por las fuerzas de la oferta y la demanda y daba por ende inestabilidad y alzas abusivas de los precios tanto de insumos como del producto final, debido a las diferencias de los niveles de producción que tiene el mercado nacional en comparación con los internacionales, obligando a que dicho mercado haya requerido la intervención del Estado.

La intervención que se da por medio de las reformas anteriormente mencionadas, a través de lo que se conoce como precios de sustentación (precios mínimos); mecanismo que permitía una estabilidad relativa a productores y consumidores. Igualmente se conservaban las políticas de abastecimiento reguladas a través del comercio exterior o almacenamiento y formación de inventarios estratégicos con la compra y venta del arroz a cargo de diferentes instituciones del Estado.

Simultáneamente a la situación nacional, países latinoamericanos como Uruguay y Brasil, ante la apertura total de los mercados, se encontraban desarrollando estrategias en sus respectivos mercados internos, con el objeto de mejorar la competitividad de sus productos de cara a los nuevos requerimientos de los mercados internacionales; y así asegurar su sostenibilidad, dado el carácter estratégico que tiene el arroz tanto por su papel en la seguridad alimentaria, como en la generación de empleos. Ante tal coyuntura internacional, Costa Rica declaró el arroz como un producto sensible y adoptó su protección con la Ley № 7472 antes mencionada.

14 A partir de 1975, la región centroamericana experimentó un fenómeno inflacionario donde la tasa promedio de la inflación anual aumentaba al 10\% aproximadamente, como consecuencia principalmente del incremento de los precios del petróleo. 
La disminución y la descentralización de funciones del sector estatal, así como la incorporación del arroz en diversos tratados de libre comercio a nivel mundial, han forzado a Costa Rica a tener que recurrir a otras medidas que impactan a los actores involucrados en la cadena, ello debido a la importancia del arroz en la dieta del costarricense.

Dado lo anterior, el 30 de mayo del 2002 se crea la Corporación Arrocera Nacional (CONARROZ), a la que le corresponde:

Establecer un régimen de relaciones entre productores y agroindustriales de arroz, que garantice la participación racional y equitativa de ambos sectores en esta actividad económica, y además, fomente los niveles de competitividad y el desarrollo de la actividad arrocera. (Ley № 8285 Artículo 1, p. 1).

CONARROZ jugará un papel fundamental en la orientación de una estrategia de largo plazo, con el objeto de preparar al sector arrocero de cara a los actuales y futuros tratados de libre comercio. Esto le abre a la corporación la posibilidad de continuar participando como insumo de información para el Ministerio de Economía, Industria y Comercio (MEIC), en la fijación del precio al productor y en el establecimiento de los márgenes de comercialización.

También entre las funciones de la institución está intervenir en la determinación de los montos a importar en cada período, (cuando hay desabasto) y de distribuir esas importaciones entre sus asociados (fines que se le confieren en el artículo $6^{\circ}, 7^{\circ}$ y del 37 al 40 de la ley mencionada).

Tal y como se desprende de los señalamientos anteriores, una vez promovida la reforma estatal y con ello la apertura comercial, el país se ha visto en la necesidad de modificar parte de las políticas establecidas para el sector, lo que lleva a un antes y un después, evidenciando disminución del apoyo al sector en términos de protección y garantías por parte del gobierno.

Se ha dado una transición en materia de política pública desde un fuerte intervencionismo estatal hacia una economía más abierta, donde destaca la entrada en vigencia del CAFTA en el 2008, el cual pautó una cuota libre de arancel de 50000 TM de arroz en granza con 2\% de crecimiento, mientras que para el arroz pilado se acordó 5000 TM con 5\% de crecimiento; asimismo se negoció una desgravación del arancel en $20 \%$, donde en los primeros 10 años no se desgrava, a partir del año 11 al año 15 el arancel se desgrava 40\% (proceso que inicia a partir del 2016) y del año 16 en adelante se desgrava el 60\% restante. Por otra parte, es importante recalcar que se autorizó un volumen salvaguardia de activación del 10\% del volumen de la cuota.

Es claro que dicha apertura promueve la liberalización del precio del arroz que a pesar de ser una práctica que caracteriza la globalización, se intenta resguardar al sector implementando mecanismos que favorezcan la producción y permitan garantizar la seguridad alimentaria de los sectores menos favorecidos. 


\section{Discusión}

El análisis pormenorizado de las condiciones en las que se desenvuelve el mercado arrocero en Costa Rica, permite extraer algunas valoraciones importantes a saber:

- La producción nacional de arroz ha sido fluctuante debido principalmente a factores climáticos y a la reconversión productiva de ciertas áreas, dentro de las cuales destaca la Región Chorotega; esta situación no solo se evidencia en la producción, sino también en el rendimiento y en el aumento de la dependencia del mercado internacional, con la salvedad del periodo 2009-2010, cuando se dio una sobreproducción que limitó la importación del grano.

- Tal condición se dificulta en una economía que busca el libre intercambio, ya que con la firma de tratados de libre comercio y la desgravación arancelaria pactada se toman medidas contradictorias, lo que redunda negativamente en el sector, por lo que se vuelve imperativo tomar acciones o decisiones de política pública, centradas en mejoras de los sistemas de producción nacional mediante capacitaciones, asociaciones, mejor infraestructura y paquetes tecnológicos principalmente, que permitan crear las condiciones para que los productores puedan enfrentar la apertura.

- Si no se actúa en favor de los productores nacionales, muchos de estos se verán obligados a dejar la actividad, lo que impactará negativamente el empleo, los ingresos y por consiguiente se puede dar un aumento de la pobreza, resaltando que estos productores habitan en zonas que poseen tasas de desempleo y de pobreza tanto no extrema como extrema por encima del promedio nacional.

- De igual forma, dicha afectación no se dará solo a nivel de productores ya que el arroz es el principal alimento dentro de la dieta de los costarricenses, especialmente dentro de los quintiles de menor ingreso, cuya cantidad de miembros es alrededor de cuatro por hogar.

- El desarrollo de los coeficientes de dependencia y autosuficiencia alimentaria, permiten evidenciar la sensibilidad del sector a las fluctuaciones de los mercados externos; previo a la crisis del 2008-2009 las importaciones de arroz aumentaron, lo que se tradujo en una mayor dependencia de dicho grano; sin embargo, esta tendencia cambió para los siguientes periodos de crisis, alcanzando en 2010-2011 un nivel de dependencia de tan solo en 10,28\% del arroz importado. Pese a ello, la situación se revertió y cada vez se depende más de las importaciones; para el período 2012-2013 alcanza 37,25\%. Ello aunado al perjuicio para la gran cantidad de micro y pequeños productores que dependen absolutamente de dicha actividad.

- El ligamen directo con los precios internacionales convierte al país en una economía susceptible a las fluctuaciones del mercado internacional, situación poco deseable ya que ante presiones en los mercados internacionales se puede afectar el consumo, la producción de arroz y el acceso a este grano. 
- Las condiciones actuales del mercado arrocero en Costa Rica, aunadas a las tendencias globalizantes y de libre comercio, convierten al sector arrocero en un eje fundamental de atención en Costa Rica, particularmente porque es el principal producto dentro de la canasta básica alimentaria, con un consumo promedio per cápita para el período 2000-2013 de $51,78 \mathrm{~kg}$.

- El Estado desde los años sesenta ha intervenido directamente en el mercado arrocero mediante determinadas políticas públicas como la creación de la Ley № 7472 sobre la Promoción de la Competencia y Defensa Efectiva del Consumidor. La decisión se tomó al considerar al sector como oligopólico; por tanto, procuran controlar posibles prácticas desleales en el mercado.

Finalmente, el análisis realizado permite reconocer la importancia del sector arrocero costarricense en el tema de seguridad alimentaria, en donde la volatilidad de los precios no solo puede causar efectos en el corto plazo. Se debe trabajar tanto para mejorar su competitividad (crecimiento económico) como para garantizar a la población una oferta de arroz de calidad que contribuya con una mejora de la calidad de vida de los habitantes (desarrollo económico).

\section{Referencias bibliográficas}

Baptiste, B. (2007). Liberalización comercial y producción de arroz en Haití. Observatorio de la Economía Latinoamericana № 87.

Bautista, L. R. (Marzo, 2004). Claridades Agropecuaras. Apoyos y Servicios a la Comercialización Agropecuaria , 51.

Combbes, J. y. (2002). ¿Cómo influye la apertura comercial en los déficits presupuestarios de los países en desarrollo? París: $51^{\circ}$ Congreso de la Asociación Francesa de Ciencias Económicas (AFSE).

Corporación Nacional Arrocera (CONARROZ). Datos Estadísticos de varios años.

IICA, I. I. (2012). Situación de la seguridad alimentaria en las Américas. San José, Costa Rica.

Faletto, C. (1977). Dependencia y desarrollo en América Latina.

Instituto Nacional de Estadística y Censos (INEC). Estadísticas Económicas. 
Ley 7472, Ley para la Promoción de la Competencia y Protección efectiva del Consumidor. Entrada en vigor: 19 de enero de 1995.

Ley 8285, Ley de Creación de la Corporación Nacional Arrocera. Entrada en vigor 14 de junio de 2002.

Organización de las Naciones Unidas para la Alimentación y Agricultura. 2010. Seguimiento del Mercado del arroz. Tomada de http://www.fao.org/docrep/012/al186s/al186s00.pdf

Oriza. 2012. El consumo del arroz per cápita está aumentando en los países desarrollados, según la FAO. Tomado de http://arroz.com/content/el-consumo-de-arroz-c\%C3\%A1 pitaest\%C3\%A1-aumentando-en-los-pa\%C3\%ADses-desarrollados-seg\%C3\%BAn-la-fao

Rubio, A. (2003). Los impactos socioeconómicos y ambientales de la liberalización comercial de los granos básicos en el contexto del TLCAN: El caso de Sinaloa. México: Centro de Estudios para el Cambio en el Campo Mexicano . 